\title{
Research on water-line corrosion of carbon steel by wire beam electrode technique
}

\author{
Yalin Chen ${ }^{1,2, a}$, Wei Zhang ${ }^{1,2}$, Jian Ding ${ }^{3}$, Pengfei Yin ${ }^{1,2}$, Jia Wang ${ }^{3}$ and Yan Zou ${ }^{4}$ \\ ${ }^{1}$ Qingdao NCS Testing \& Corrosion Protection Technology Co., Ltd., 266071 Qingdao, China \\ ${ }^{2}$ Qingdao Research Institute for Marine Corrosion, 266071 Qingdao, China \\ ${ }^{3}$ College of Chemistry and Chemical Engineering, Ocean University of China, 266100 Qingdao, China \\ ${ }^{4}$ Institute of Oceanographic Instrumentation, Shandong Provincial Key Laboratory of Ocean Environment Monitoring Technology, 266100 \\ Qingdao, China
}

\begin{abstract}
The wire beam electrode (WBE) method was employed to study water-line corrosion of carbon steel under static condition. It was found that, it was the thin electrolyte film that aggravated the corrosion of steel in water-line area, not the difference of oxygen concentration. The difference of oxygen concentration decided the location of cathode, which was near waterline, and the regularity of vertical current distribution under waterline. Besides, it was also found that, in water-line area, the peak of corrosion rate was located at about half of the region just below the waterline.
\end{abstract}

\section{Introduction}

The air-liquid-solid interface is the area where heavy corrosion always happens. This kind of corrosive phenomenon is widely found. The factors influence it are multitudinous, so more and more researchers focus on the study of water-line corrosion.

The first scholar that illustrated the water-line corrosion was Evans[1,2]. After research, it was found that the carbon steel, which was partly immersed in $\mathrm{KCl}$ electrolyte, had heavy local corrosion. The part under waterline corroded heavily, but right near the waterline, the metal hardly corroded. He thought the area near airliquid interface was full of dissolved oxygen but the area under waterline was comparatively short of dissolved oxygen. Jeffrey[3,4] studied the corrosion of long vertical steel strips in the marine tidal zone. It showed that the steel right near waterline had slightly corroded, the heaviest corrosion happened about at $250 \mathrm{~mm}$ above the waterline and $200 \mathrm{~mm}$ below the waterline. Jeffrey thought it was the alternant distribution of cathode and anode.

The results described above were got from corrosion morphology and mass loss. For lack of in-suit data about electrochemistry, it was unconvincing to speculate the distribution of cathode and anode. The data of one steel strip was homogenized by conventional method. So it was difficult to observe the electrochemical information about localized corrosion reaction, which was crucial to reveal the corrosion mechanism in water-line area.

The Wire Beam Electrode (WBE) is fabricated from several short connected wire electrodes regularly arranged as a matrix. For each wire electrode is very

\footnotetext{
a Corresponding author: stcyl163@163.com
}

small compared with the whole WBE, the electrochemical reaction on a wire electrode is regarded as being homogeneous[5,6]. The WBE method plays an important role in studying localized corroded metal surface. W. Wei[7] studied the electrochemical behavior at biofilm/stainless steel interface by the WBE. It was turned out that the heterogeneity of biofilm had a close relationship with the local potential and current on.

In this paper, our study was to reveal the water-line corrosion mechanism of carbon steel in static 3.5\% NaCl solution by the WBE technique. Through comparing the two WBEs of different sizes, we wished to have in-depth knowledge of kinetics of accelerated corrosion in waterline area.

\section{Experimental}

The small WBE was fabricated from 121 Q235 wires regularly arranged as a $11 \times 11$ matrix ( $1.6 \mathrm{~mm}$ diameter), embedded in epoxy resin with an interval of $1 \mathrm{~mm}$ from each other, as shown in Fig 1a. The big WBE was fabricated from 120 Q235 square pieces regularly arranged as a $6 \times 20$ matrix ( $1 \mathrm{~cm}$ side length), embedded in epoxy resin with an interval of $1 \mathrm{~cm}$ from each other, as shown in Fig 1b. Before starting immersion, the WBEs were polished with metallurgical paper up to no.1000 grade, and cleaned with acetone. The $3.5 \% \mathrm{NaCl}$ solution(mass to volume ratio) was applied as electrolyte. The waterline located at Row3 of the small WBE and Row5 of the big WBE. 


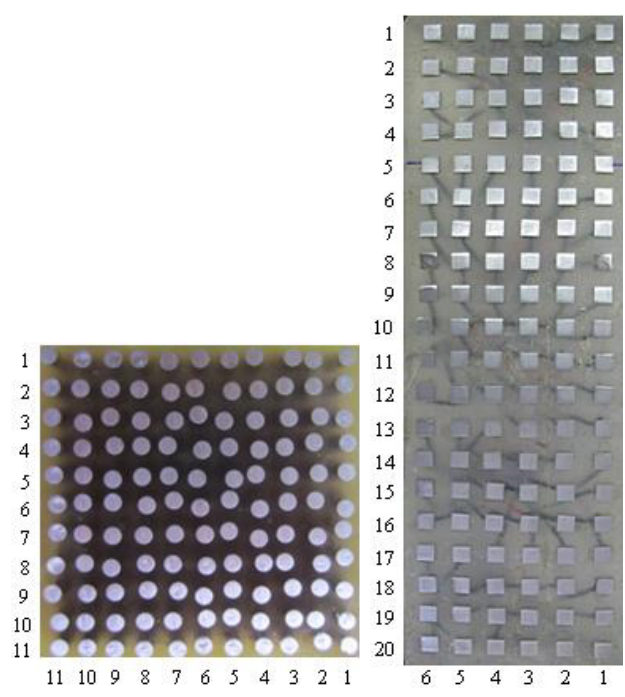

Figure 1. Photos of the small WBE surface(a) and the big WBE surface(b)

The testing device for current distribution was developed by our laboratory[8]. The current of each microelectrode in the WBE was measured one by one. When the test began, the No.1 microelectrode was disconnected from the other 120 shorted microelectrodes and the galvanic current between the No.1 microelectrode and the other 120 shorted microelectrodes were measured[9]. The current distribution was mapped with Surfer 8.0 software. In the current distribution map, the ticks in $\mathrm{x}$ and $\mathrm{y}$ axis were column and row number of the microelectrode in the WBE, respectively.

\section{Result and discussion}

Fig 2 showed the evolution of current density distribution of the small WBE surface after various immersed time. The current unit is $\mu \mathrm{A} \cdot \mathrm{cm}-2$, the cathodic current is negative and the anodic current is positive. The blue represented cathodic current, the red represented anodic current.

To describe the vertical distribution of current density, single row current density was put forward. Single row current density was obtained by dividing the current sum of 11 wires in one row by the area of the 11 wires. Fig 3 were the changes in vertical current density, corresponding to the different immersion time of fig 2 .

As shown in fig $2 \mathrm{a}$ and $3 \mathrm{a}$, at the beginning of immersion, the surface of WBE did not showed centralized anode region or cathode region. After immersion for 12 hours, the cathode region was mostly being concentrated in Row3 and 4(fig $2 \mathrm{~b}$ and fig $3 \mathrm{~b}$ ). The current value was larger than that after immersion for 2 hours. The peak of cathode current was located in Row3(fig 2b).

On the surface of Row1 and 2, liquid gradually took the place of gas. As a result, the surface of Row1 and 2 was covered with thin electrolyte film[10]. In thin electrolyte layer, the diffusion rate of oxygen was very high, further, the cathodic reaction rate was very high[11]. So the WBE partly submerged became an oxygen concentration cell. That is why Row 1 and 2 showed cathode current. But at the initial stage of the trial, as the film was too thin, the reaction resistance was very high[12]. The Row3 was located at the boundary between gas and liquid, the cathodic reaction happened on Row3 would react with lower resistance. So the cathode current of Row3 was larger than that of Row1 and 2. Row4 and 5 were closer to waterline, the dissolved oxygen concentration was larger than that of Row6 to 11 . So the region where was relatively rich in $\mathrm{O} 2$ became cathode region and the region where was relatively poor in $\mathrm{O} 2$ became anode region[13].
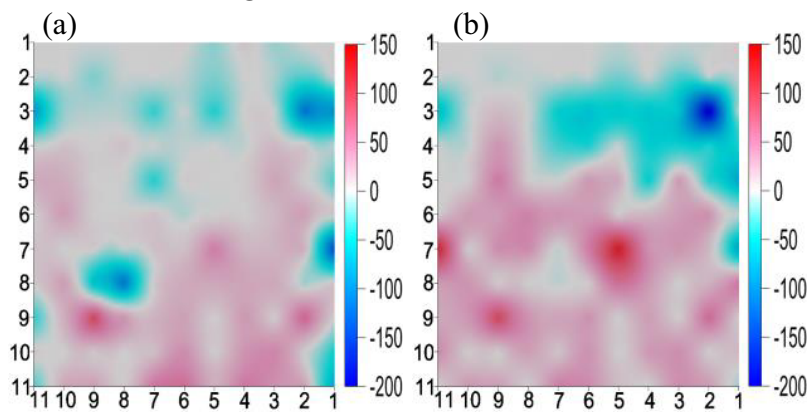

(c)

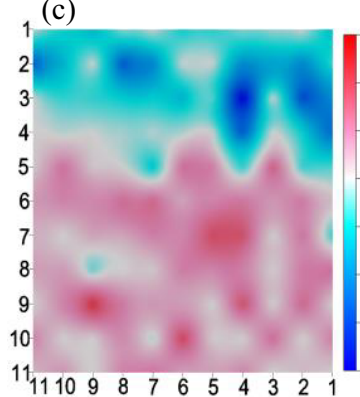

(d)

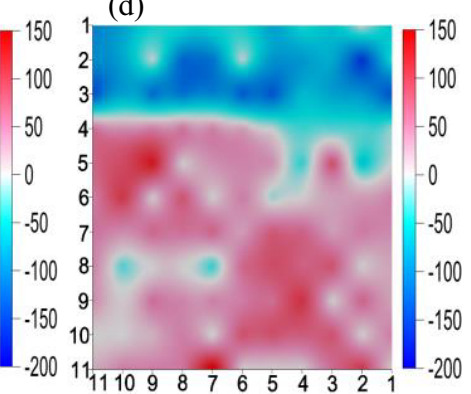

(e)

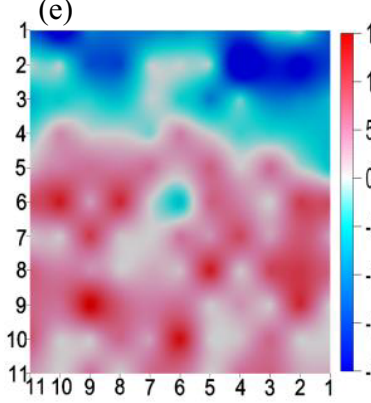

(f)

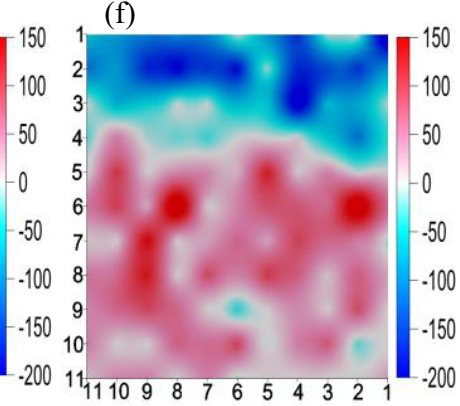

Figure 2. Evolution of current density distribution with immersion time $2 \mathrm{~h}(\mathrm{a}), 12 \mathrm{~h}(\mathrm{~b}), 2 \mathrm{~d}(\mathrm{c}), 6 \mathrm{~d}(\mathrm{~d}), 18 \mathrm{~d}(\mathrm{e}), 40 \mathrm{~d}(\mathrm{f})$

After immersion for 2 days, the current got larger(fig 2c). Meanwhile, the cathode region became the first 4 rows (fig 3c). The cathodic current value of Row1 and 2 increased a lot, especially Row2.

The increase of cathodic current of Row1 and 2 indicated the thin electrolyte film was getting thicker. When the cathodic reaction above the waterline sped up, below the waterline, the anodic reaction would sped up and the cathodic reaction rate would reduce. This was why the current polarity of Row 5 became anodic. From the phenomenon, it could be seen that it was the growth of thin electrolyte film above waterline that accelerated the anodic reaction below the waterline.

After immersion for 6 days, the current increased further. (fig 2d), the current polarity of Row4 was also reversed, the cathode region became Row 1 to 3 and the anode region became Row4 to 11( fig 3d). 


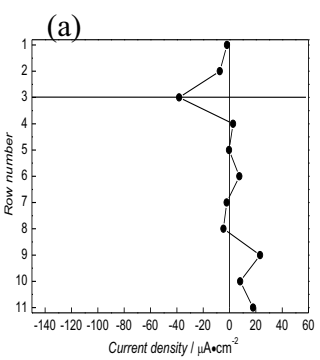

(b)

(c)
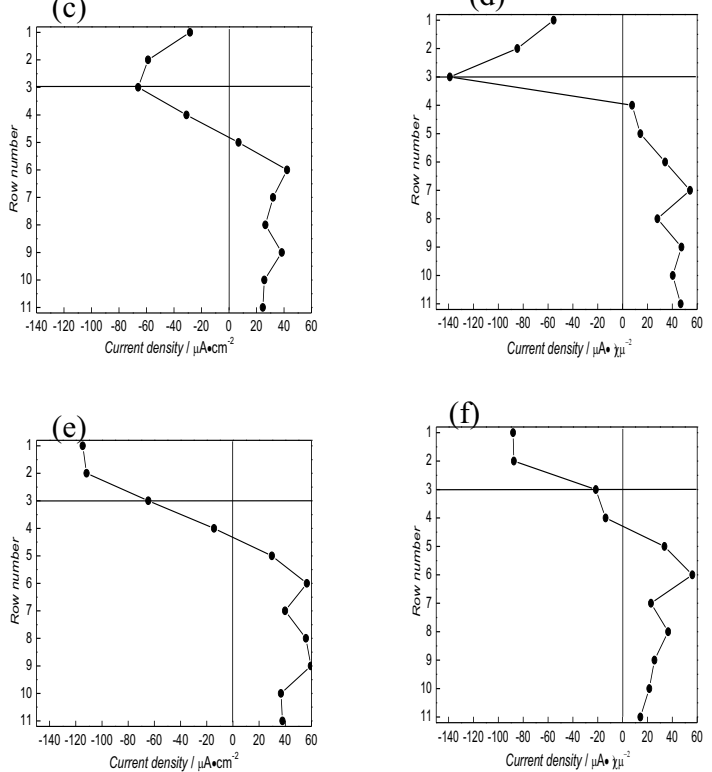

Figure 3. Variations of current density in vertical direction after immersion for $2 \mathrm{~h}(\mathrm{a}), 12 \mathrm{~h}(\mathrm{~b}), 2 \mathrm{~d}(\mathrm{c}), 6 \mathrm{~d}(\mathrm{~d}), 18 \mathrm{~d}(\mathrm{e}), 40 \mathrm{~d}(\mathrm{f})$

The reason why the current polarity of Row4 occurred reversal was similar to that of Row5, it also indicated that the thickness of thin electrolyte film increased further. But the thickness of thin electrolyte film restricted the cathodic reaction capability of Row 1 and 2, so the cathode current of Row3 became larger.

The current distribution, as shown in fig $2 \mathrm{~d}$, lasted to the 18th day. After that, the cathodic current of Row 1 and 2 got further large(fig 2e) and went over that of Row3(fig $3 \mathrm{e})$. The current polarity of Row4 was reversed again, and the cathode region became Row 1 to 4 . Form the 18 th day on, the current distribution did not change a lot any more.

In the first 6 days, the anodic current increased with the increase of cathodic current of Row 1 and 2. Which meant it was the growth of thin electrolyte film that aggravated the corrosion under waterline. However, the cathodic current of Row3 also kept increasing in the first 6 days. So it was necessary to know if the Row3 had effect on accelerating corrosion under waterline. The liquid film on the upper half of Row3 was formed by surface tension, so the film was thick and the thickness of diffusion layer could change little. That was to say under the condition of constant environment, it was impossible for Row3 to aggravate corrosion(the following contrast trial proved this view). Under the waterline, the anodic current was increasing gradually, that would result in decrease of cathodic current[14]. So the increase of cathodic current of Row3 should be caused by the decrease of cathodic current under the waterline. However, the cathodic reaction rate of Row1 and 2 was limited by thickness of thin electrolyte film, so the cathodic current of Row3 was the highest.

In the early days of the trial, it showed the cathodic reaction in thin electrolyte film had high resistance. The resistance mainly came from the hydration of ion[15]. Since the ion participated in cathodic reaction was in the form of hydrated ion, the amount of $\mathrm{H} 2 \mathrm{O}$ affected the resistance of hydration, and even the transfer resistance of hydrated ion. When the film was thick enough, the resistance of hydration could be ignored. So from the 18th day on, Row 1 and 2 became the main cathode region.

Fig 4 showed the variations of anodic current density of the WBE with immersion time. The anodic current density was obtained by dividing the anodic current sum by the area of WBE. If the cathodic reaction in anode region was ignored, the anodic current density could represent the corrosion rate approximately.

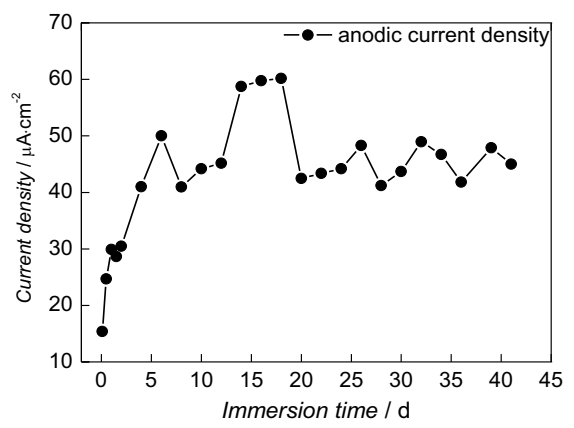

Figure 4. Variations of anodic current density of the WBE with immersion time

As shown in fig 4, in the first 18 days, the corrosion rate increased gradually, and then it dropped and went to stabilization. It was noted that, as known from other studies, in fully immersed zone[16], the corrosion rate would get down with the accumulation of rust. But in the water-line zone, the corrosion rate kept going up until the 18 th day of the trial. It indicated that there should be a factor that had effect on aggravating corrosion. From the above analysis, only the thin electrolyte film, essentially the variation of thickness of the film, had the effect.

To confirm if the thin electrolyte film have the effect on aggravating corrosion and if the size of WBE affect the current distribution under waterline, a bigger WBE was employed in a comparative trial. The same experiment method and data processing method were applied to the comparative trial. Fig 5 was the evolution of current distribution of the big WBE and fig 6 was the changes in vertical current density, corresponding to the different immersion time of fig 5 .

In the first 12 days(fig $5 \mathrm{a}-5 \mathrm{c}$ ), the evolution of current distribution was similar to that of small WBE. With the immersion time going, the cathode region was being concentrated, and then the cathode region got shrunk toward the waterline. As shown in fig $6 \mathrm{~b}$ and $6 \mathrm{c}$, on the 5th day, the cathode region was from Row5 to Row7, then on the 12th day, the cathode region became Row5. The cathodic current density of Row5 was always 
the highest. As the big WBE was also an oxygen concentration cell, the big WBE and small WBE had some features of oxygen concentration cell in common.

But compared with the small WBE, the big WBE also showed some distinctions in evolution of current distribution. Firstly, there was always no obvious current above the waterline. Secondly, the anodic current density decreased gradually in the first 12 days. It indicated that, the pieces under thin electrolyte film above waterline did not participated in the reaction of oxygen concentration cell.

On the 25th day, the used electrolyte was replaced with fresh. Immediately, to the 26th day, the current distribution changed a lot. The current density rose sharply(fig 5d), and the cathode region became Row5 to 10 (fig 6d). The reason was that there was more dissolved oxygen in the new electrolyte. For the small WBE, the effect of environment could be ignored compared with the effect of thin electrolyte film. But for the big WBE, there was no factor to aggravate corrosion except the corrosion environment. This also confirmed that the film created by surface tension had no effect on aggravating corrosion
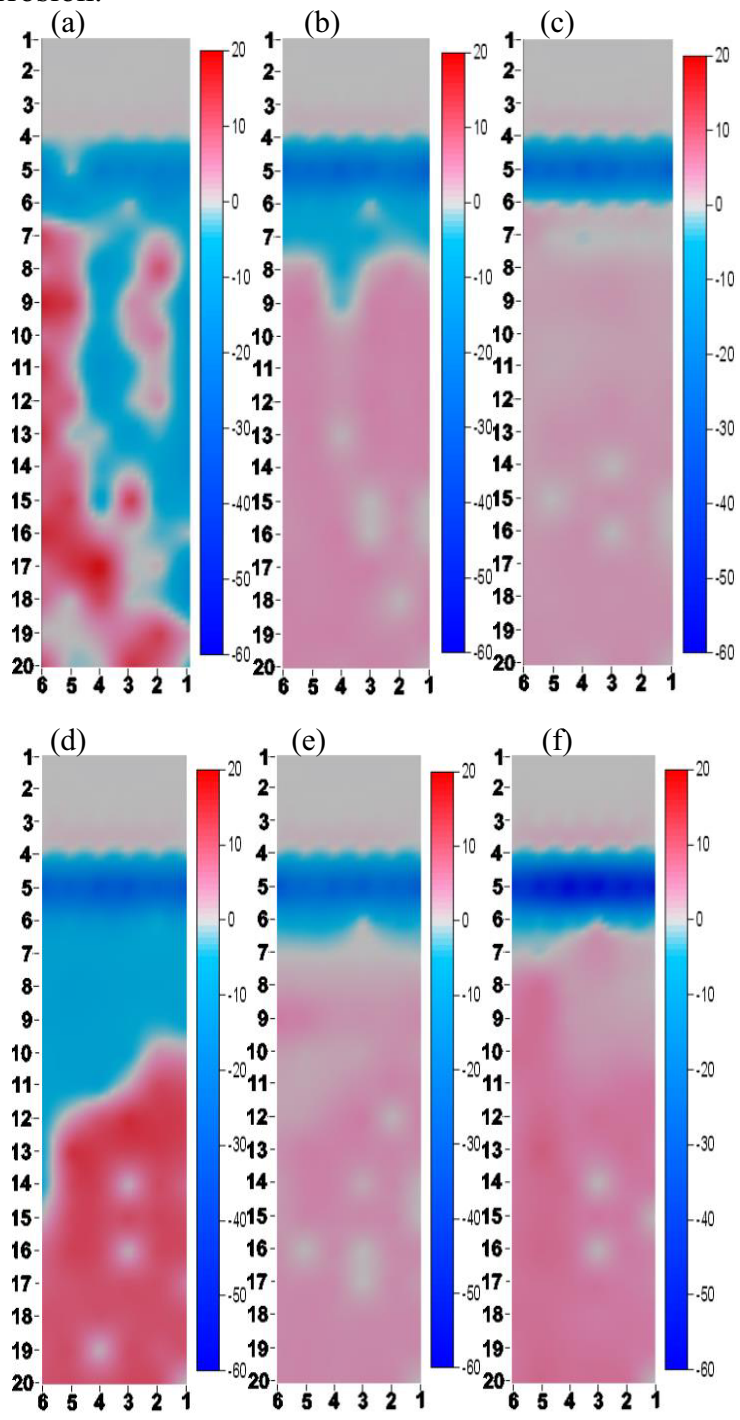

Figure 5. Evolution of current density distribution with immersion time $2 \mathrm{~h}(\mathrm{a}), 5 \mathrm{~d}(\mathrm{~b}), 12 \mathrm{~d}(\mathrm{c}), 26 \mathrm{~d}(\mathrm{~d}), 37 \mathrm{~d}(\mathrm{e}), 58 \mathrm{~d}(\mathrm{f})$
With the consumption of dissolved oxygen and the accumulation of corrosion products, the anodic current density decreased then(fig $5 \mathrm{e}$ ). On the 37 th day, the current distribution returned to that of 12 days ago, like fig 5c. The cathode region was mainly located in Row5 to 6(fig 6e). From then on, the current distribution did not have obvious change(fig $5 \mathrm{f}$ and $6 \mathrm{f}$ ).

Fig 7 was the variations of anodic current density with immersion time, it was obviously contrary to fig 4 . Without considering the effect of replacing electrolyte, the trend of variations was that, it got to peak quickly and then decreased sharply, from the 5th day on, it went to stabilization(the fluctuation range was less than $1 \mu \mathrm{A} \cdot \mathrm{cm}-$ $2)$. The trend of variations was similar to that in fully immersed zone. This illustrated the corrosive condition of the big WBE was fairly stable, though it was an oxygen concentration cell, without thin electrolyte film, the corrosion could not be aggravated.
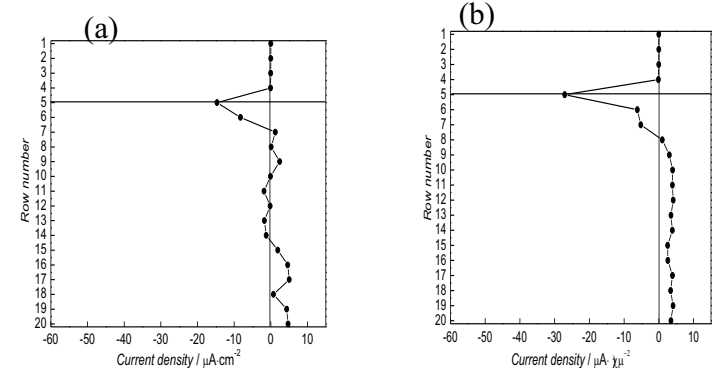

(c)
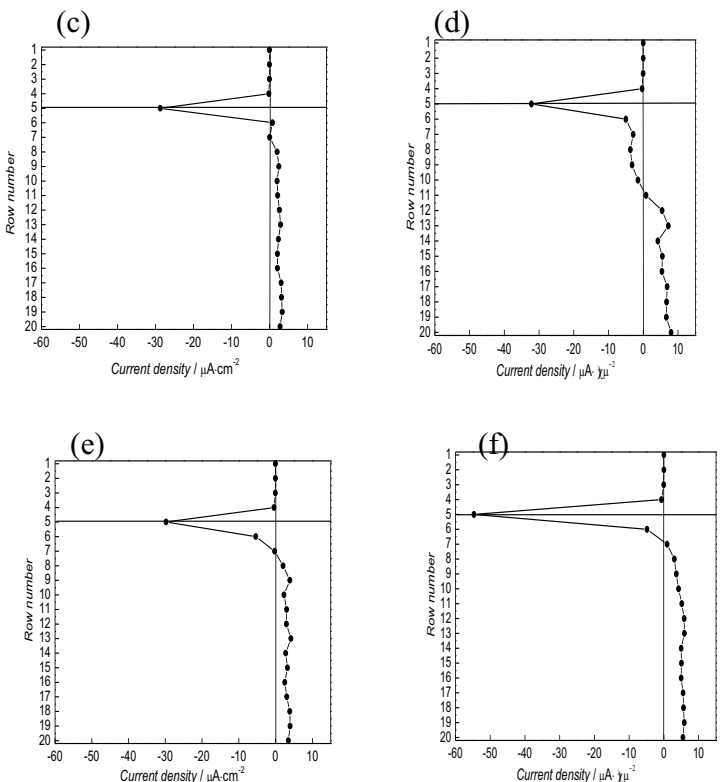

Figure 6. Variations of current density in vertical direction after immersion for $2 \mathrm{~h}(\mathrm{a}), 5 \mathrm{~d}(\mathrm{~b}), 12 \mathrm{~d}(\mathrm{c}), 26 \mathrm{~d}(\mathrm{~d}), 37 \mathrm{~d}(\mathrm{e}), 58 \mathrm{~d}(\mathrm{f})$

The two WBEs respectively represented the oxygen concentration cell with and without thin electrolyte film. The similarities between the small WBE and the big one showed the features of oxygen concentration cell. For example, the cathode was located near the waterline and the lower part of the WBE corroded faster than the part near the waterline. But the oxygen concentration cell itself could not aggravate corrosion under waterline. From the above trials, it could be seen the corrosion rate increased only with the growing of thin electrolyte film 
under static condition. Besides, with thin electrolyte film, the corrosion rate would stabilize at a higher level than the original value, without it, the corrosion rate would stabilize at lower level. In fact, it was the thickness of thin electrolyte film that decided the thickness of diffusion layer, and then affected the diffusion rate of oxygen[17,18]. The diffusion rate of oxygen affected the cathodic reaction rate and then the anodic reaction rate.

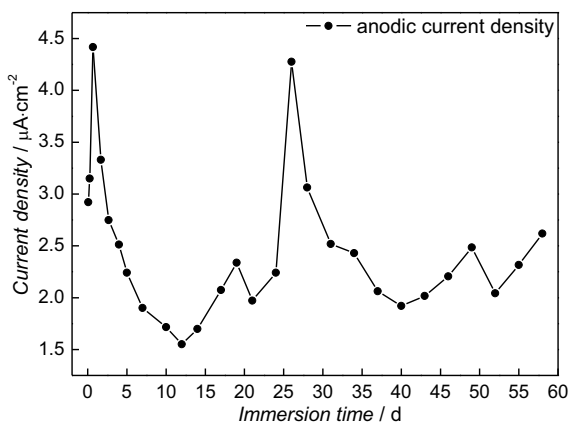

Figure 7. Variations of anodic current density of the WBE with immersion time

In addition, after calculating the average current density of each row during the whole immersion period, (The average current density was obtained by adding the current densities of one row measured every two days and then averaging the sum.) it was found that, under waterline, the variations of vertical current density of the two WBEs showed the same regularity, as shown in fig 8 . The average current density could approximately show the corrosion status of the row.
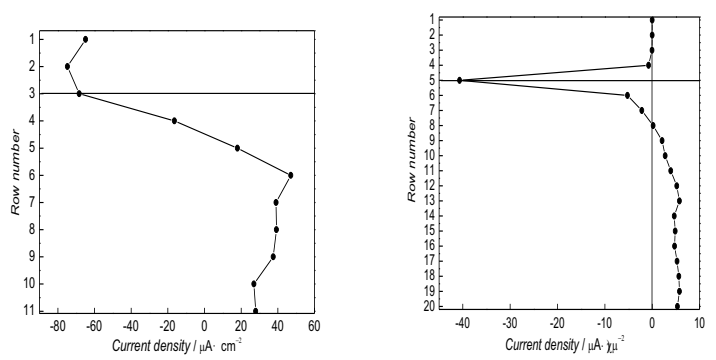

Fig 8. The vertical variations of current density of the small WBE(a) and the big WBE(b)

Under waterline, with the increase of immersed depth, for the small WBE, the corrosion rate increased rapidly until Row 6, and then decreased gradually, and for the big WBE, the corrosion rate increased gradually until Row 13, and then decreased slightly to a stable level. The distance from waterline to the peak of corrosion rate was $7.8 \mathrm{~mm}$ and $17 \mathrm{~cm}$ respectively. In the view of relative position of corrosion region (corrosion region was the region just below the waterline and covered with rust), the peak of corrosion rate of the small WBE was located at $4 / 9$ of the corrosion region, and for the big WBE, it was located at $9 / 16$ of the corrosion region. Whether thin electrolyte film affected corrosion or not, both the peaks of corrosion rate were located at near half of the respective corrosion regions. So it was conjectured that, in the water-line zone, the peak of corrosion rate was located at near half of corrosion region under static condition.

\section{Conclusion}

The WBE method was employed to investigate the waterline corrosion of steel under static condition. From the above two trials, the following conclusions were obtained:

(1) Under static condition, it was the thin electrolyte film that aggravated the corrosion of steel in water-line area, not the difference of oxygen concentration. As the thickness of thin electrolyte film increased, the corrosion rate increased first, and then stabilized at a very higher level than the original value. The difference of oxygen concentration decided that the cathode was located near waterline, and decided the regularity of vertical current distribution under waterline.

(2) When the oxygen concentration cell in water-line area did not have thin electrolyte film, the corrosion rate decreased sharply from origin, and then stabilized at a low level. So for anti-corrosion of steel in water-line area, it was effective to make the circuit between the thin electrolyte film and the solution below break.

(3) In water-line area, the peak of corrosion rate was located at about half of the region just below the waterline.

\section{Acknowledgments}

This work is supported by NSFC (No.21203034; No.51209129; No.51131005).

\section{References}

1. U.R. Evans, Report on corrosion research work at Cambridge University interrupted by the outbreak of war, J. Iron. Steel. Inst. 141, 219-234 (1940)

2. U.R. Evans, The Corrosion and Oxidation of Metals (Edward Arnold, London, 1960)

3. R. Jeffrey, R.E. Melchers, Corrosion of vertical mild steel strips in seawater. Corros. Sci. 51, 2291-2297 (2009)

4. R.E. Melchers, R. Jeffrey, Corrosion of long vertical steel strips in the marine tidal zone and implications for ALWC. Corros. Sci. 65, 26-36 (2012)

5. Y. J. Tan, Wire beam electrode: a new tool for studying localised corrosion and other heterogeneous electrochemical processes, Corros. Sci. 41, 229-247 (1998)

6. X. Zhang, W. Wang, J. Wang, A novel device for the wire beam electrode method and its application in the ennoblement study, Corros. Sci. 51, 1475-1479 (2009)

7. W. Wang, X. Zhang, J. Wang, The influence of local glucose oxidase activity on the potentialrrent distribution on stainless steel: a study by the wire beam electrode method, Electrochim. Acta 54, 55985604 (2009)

8. Y.J. Tan, S. Bailey, B. Kinsella, Mapping nonuniform corrosion using the wire beam electrode 
method. III. Water-line corrosion, Corros. Sci. 43, 1931-1937 (2001)

9. Y. H. Wang, W. Wang, Y. Y. Liu, L. Zhong, J. Wang, Study of localized corrosion of 304 stainless steel under chloride solution droplets using the wire beam electrode, Corros. Sci. 53, 2963-2968 (2011)

10. Z.J. Liu, W. Wang, J. Wang, Study of corrosion behavior of carbon steel under seawater film using the wire beam electrode method, Corros. Sci. 80, 523-527 (2014)

11. M. Stratmann, The investigation of the corrosion properties of metals covered with adsorbed electrolyte layers-A new experimental technique, Corro. Sci. 27, 869-872 (1987)

12. Mattsson E, Corrosion: an electrochemical problem, Chemical Technology 4, 234 235 (1985)

13. N.D. Tomashov, Theory of Corrosion and Protection of Metals (MacMillan, New York, 1966)

14. A. Nishikata, Y. Ichihara, Y. Hayashi, T. Tsuru, Influence of electrolyte layer thickness and $\mathrm{pH}$ on the initial stage of the atmospheric corrosion of iron, J. Electrochem. Soc. 144, 1244-1252 (1997)

15. C.N. Cao, Principles of Electrochemistry of Corrosion (Chem. indus. P. Beijing, 2008)

16. M. S. Venkatraman, I. S. Cole, B. Emmanuel, Model for corrosion of metals covered with thin electrolyte layers: Pseudo-steady state diffusion of oxygen, Electrochim. Acta 56, 7171-7179 (2011

17. J. Wang, T. Tsuru, An investigation on oxygen reduction under thin electrolyte layer using Kelvin probe reference electrode, J. Chin. Soc. Corros. Pro. 17, 9-13 (1997)

18. J. Wang, T. Tsuru, Electrochemical measurements under thin electrolyte layer using Kelvin probe reference electrode, J. Chin. Soc. Corros. Pro. 15, 173-179 (1995) 